\title{
The physics of electron internal transport barriers in the TCV tokamak
}

\author{
S. Coda, E. Asp, E. Fable, T.P. Goodman, O. Sauter, V.S. Udintsev, \\ R. Behn, M.A. Henderson, A. Marinoni, G.P. Turri, C. Zucca and \\ the TCV team
}

Ecole Polytechnique Fédérale de Lausanne, Centre de Recherches en Physique des Plasmas, Association EURATOM-Confédération Suisse, EPFL SB CRPP, Station 13, CH-1015 Lausanne, Switzerland

E-mail: stefano.coda@epfl.ch

Received 10 January 2007, accepted for publication 9 May 2007

Published 27 June 2007

Online at stacks.iop.org/NF/47/714

\begin{abstract}
Electron internal transport barriers (eITBs) are generated in the TCV tokamak with strong electron cyclotron resonance heating in a variety of conditions, ranging from steady-state fully noninductive scenarios to stationary discharges with a finite inductive component and finally to transient current ramps without current drive. The confinement improvement over L-mode ranges from 3 to 6 ; the bootstrap current fraction is invariably large and is above $70 \%$ in the highest confinement cases, with good current profile alignment permitting the attainment of steady state. Barriers are observed both in the electron temperature and density profiles, with a strong correlation both in location and in steepness. The dominant role of the current profile in the formation and properties of eITBs has been conclusively proven in a TCV experiment exploiting the large current drive efficiency of the Ohmic transformer: small current perturbations accompanied by negligible energy transfer dramatically alter the confinement. The crucial element in the formation of the barrier is the appearance of a central region of negative magnetic shear, with the barrier strength improving with increasingly steep shear. This connection has also been corroborated by transport modelling assisted by gyrofluid simulations. Rational safety-factor $(q)$ values do not appear to play a role in the barrier formation, at least in the $q$ range 1.3-2.3, as evidenced by the smooth dependence of the confinement enhancement on the loop voltage over a broad eITB database. MHD mode activity is however influenced by rational $q$ values and results in a complex, sometimes cyclic, dynamic evolution.
\end{abstract}

PACS numbers: 52.55.fa, 52.25.Fi, 52.50.Sw

\section{Introduction}

Electron internal transport barriers (eITBs) [1,2] have been obtained and studied in the TCV tokamak [3] $(R=0.88 \mathrm{~m}$, $a=0.25 \mathrm{~m}, I_{\mathrm{p}}<1 \mathrm{MA}, B_{\phi}<1.54 \mathrm{~T}$, total electron cyclotron resonance heating (ECRH) power up to $4.5 \mathrm{MW}$ ) with ECRH in a variety of conditions. Fully noninductive scenarios involve an appropriate distribution of current drive (ECCD) sources sustaining a hollow current profile, further enhanced by the bootstrap current centred in the high gradient barrier region [4-7]. Depending on the details of the discharge parameters and conditions, these scenarios may or may not evolve to a true steady state, whose duration is limited merely by equipment constraints and can equal several current redistribution times and up to hundreds of electron energy confinement times. Stationary eITBs are also observed in nearly noninductive conditions, with a small Ohmic current used to fine-tune the current profile [8], as well as in discharges with comparable Ohmic and noninductive current components [9]. Finally, transient eITBs have been generated in the absence of current drive, by strong heating during current ramps. All these scenarios display a significant improvement in confinement, quantified by an energy confinement time enhancement over TCV L-mode scaling (the Rebut-Lallia-Watkins scaling [10]), $H_{\text {RLW }}$, ranging from 3 to 6 .

Although the various paths delineated above give rise to eITBs with widely varying characteristics, on average the highest performance discharges also display high bootstrap current fractions and high poloidal beta, as shown in figure 1 for a database of stationary eITBs. Higher enhancement factors have been reached in nonstationary conditions, and the bootstrap fraction has reached $90 \%$ transiently during early current ramps, as illustrated by figures 2 and 3 .

All the eITB scenarios achieved to date have relied for auxiliary heating solely on the second harmonic X-mode (X2) ECRH system, composed of six $82.7 \mathrm{GHz}$ gyrotrons delivering $0.45 \mathrm{MW}$ each to the plasma through independent 


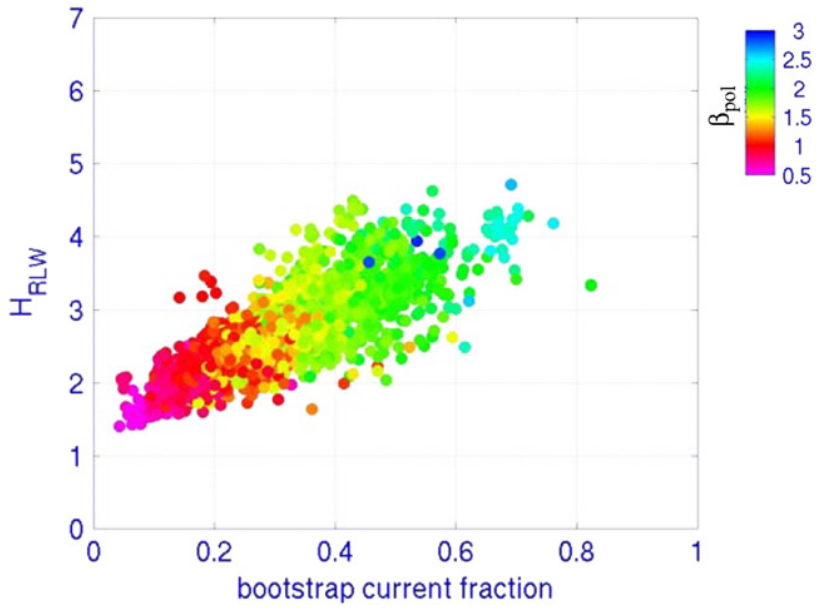

Figure 1. Electron energy confinement enhancement factor over the Rebut-Lallia-Watkins scaling [10] (TCV L-mode scaling) versus bootstrap current fraction for a database of steady-state eITBs in TCV. The colour coding refers to the poloidal $\beta$.

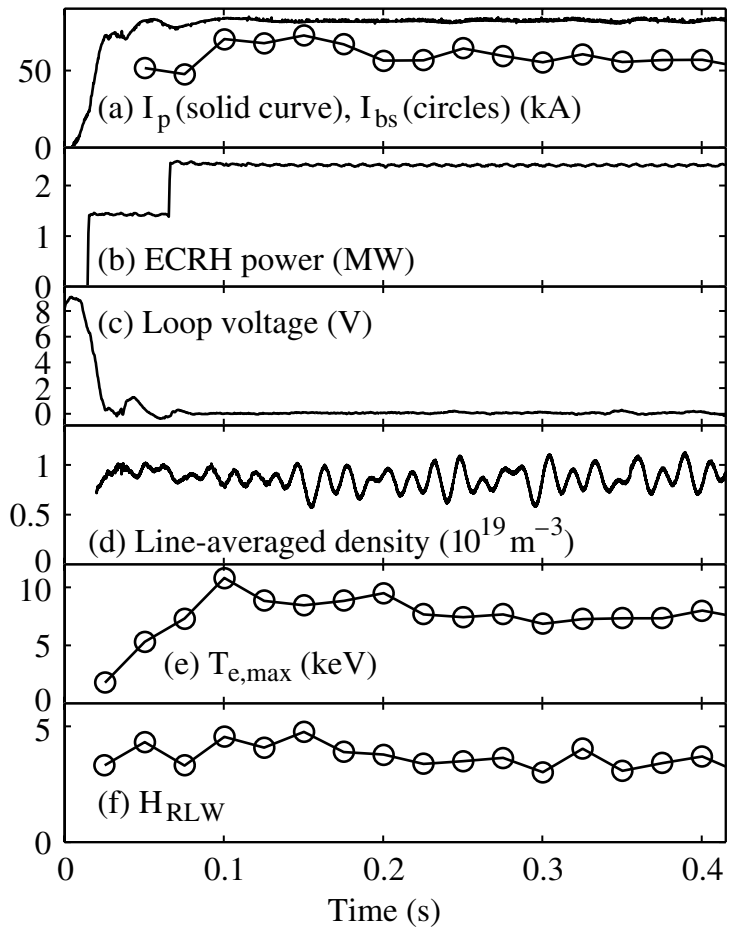

Figure 2. TCV shot 30551: the early application of ECRH power during the initial current ramp, without current drive, generates an eITB; the bootstrap fraction and the confinement enhancement factor peak at $0.15 \mathrm{~s}$ (at $90 \%$ and 4.8 , respectively); ( $a$ ) plasma current (solid curve) and bootstrap current (circles), (b) ECRH power, $(c)$ loop voltage, $(d)$ line-averaged density, $(e)$ peak electron temperature and $(f)$ energy confinement enhancement factor.

real-time-steerable launchers. The operational recipes that have been established for generating eITBs depend on the accurate positioning ability of the launchers, as the properties and dynamic evolution of the discharge have been shown to be very sensitive to the heating locations and parallel wave numbers of the various beams $[6,9,11]$. The steerability of the launchers can then be employed to control the eITB performance in dynamically varying scenarios. This has been

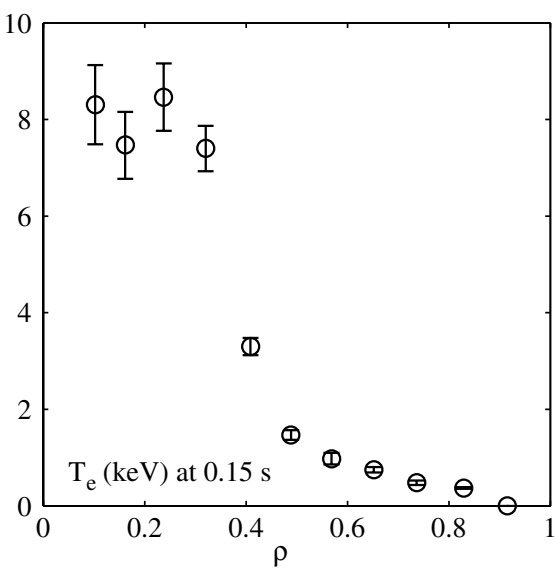

Figure 3. TCV shot 30551: the electron temperature profile at time $0.15 \mathrm{~s}$; the radial coordinate is the square root of the normalized volume.

demonstrated in recent open-loop control experiments in which the flexible plasma position and shaping control system of TCV was used to move the plasma vertically (in order to increase the effective spatial resolution of Thomson scattering measurements), and the beam aiming was pre-programmed to track the plasma displacement, keeping the eITB confinement enhancement factor constant. The control case with the beams kept fixed, in contrast, resulted in a loss of confinement [6].

\section{The role of the current density profile in eITBs}

The plasma current profile clearly plays the dominant role in determining the conditions under which a barrier can occur and also in regulating its properties and dynamic evolution. This role has been established by several dedicated studies. Since TCV lacks a direct current density measurement at present, a combination of modelling and indirect experimental measurements is used to estimate the current profile. The Ohmic and bootstrap current densities can be derived from temperature and density measurements by Thomson scattering, and the EC-driven current density is calculated by the FokkerPlanck code CQL3D [12]. The latter calculation is strongly dependent on the choice of the cross-field electron diffusivity, which in TCV regulates the ECCD broadening as well as the total driven current [13-15]. The primary constraint in the simulation is the imposition of the total EC-driven current, which is obtained by the subtraction of the Ohmic and bootstrap components from the total current. The diffusivity is adjusted so that the simulated current obeys this constraint. To perform the calculation rigorously, the computed ECCD current profile is then fed into the transport code ASTRA [16], used in the socalled diagnostic mode, in which the pressure profile is fixed (and thus the Ohmic and bootstrap currents are too) and taken from experimental data: the code calculates the total current density profile and the plasma equilibrium self-consistently, ultimately generating the safety-factor $(q)$ profile [17]. The dominant uncertainty in the procedure is the value of the effective charge $Z_{\text {eff }}$, which is difficult to determine accurately and affects significantly both the Ohmic and ECCD current estimations.

In steady-state, fully noninductive eITBs the problem is greatly simplified since the Ohmic current vanishes. These 

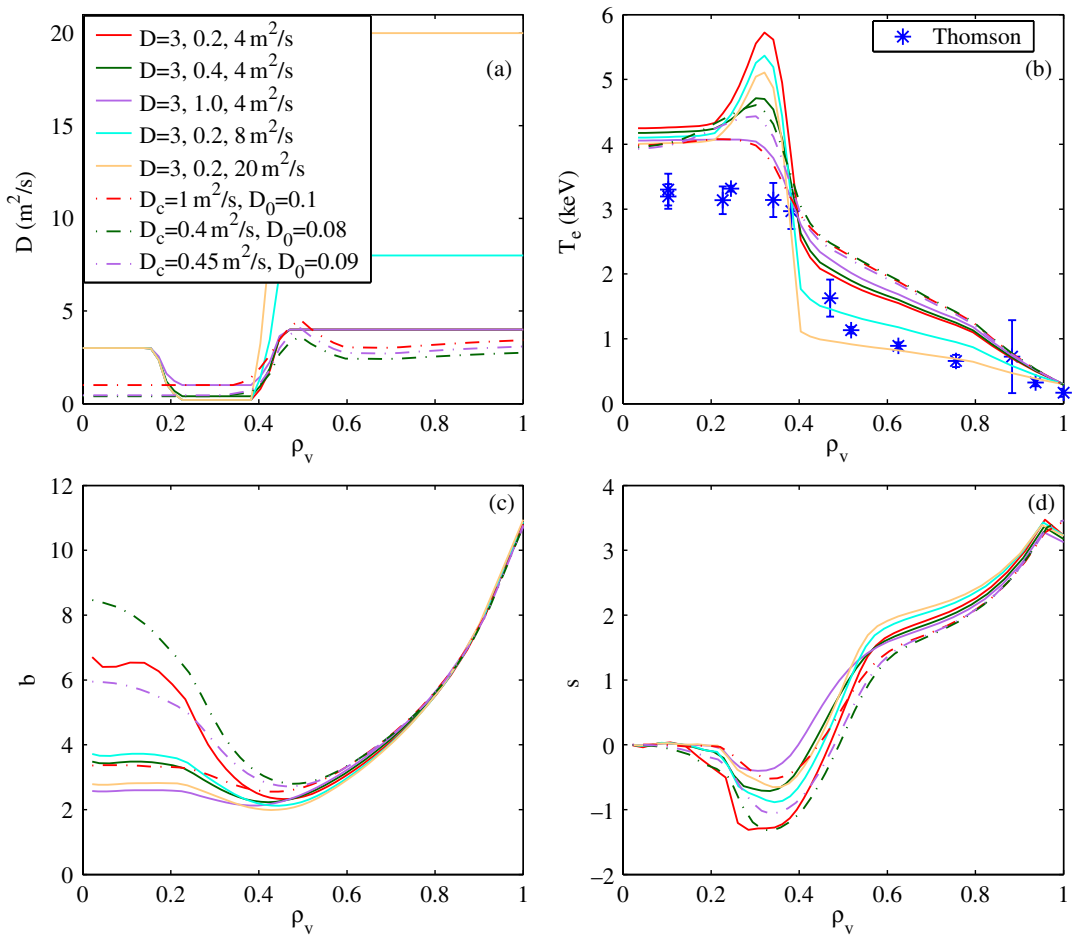

Figure 4. TCV discharge 28873: (a) electron diffusivity assumed for CQL3D simulations, $(b)$ electron temperature: CQL3D calculations versus Thomson scattering measurements, $(c)$ safety-factor and $(d)$ magnetic shear profiles calculated with the ASTRA transport code, used in diagnostic mode, with the EC-driven current provided by CQL3D. In the CQL3D runs, the electron density is constrained to match the experimentally measured profile and the diffusivities in all cases are adjusted in order to reproduce the experimentally measured EC-driven current, within the error bars. The electron temperature calculated by CQL3D includes the energy contribution from suprathermal electrons and is thus heuristically adjusted to be somewhat higher than the bulk temperature measured by Thomson scattering. The diffusivity models are as follows. Solid curves: piecewise uniform diffusivity, with the three values of the diffusivity $D$ shown in the inset corresponding, respectively, to the core region (well inside the barrier), to the power deposition region up to the point of maximum gradient, and to the region outside the barrier. Dashed curves: diffusivity proportional to power-balance energy diffusivity, with varying core values; in this case the values in the inset are the particle diffusivity in $\mathrm{m}^{2} \mathrm{~s}^{-1}$ in the region inside the ECRH deposition location, $D_{\mathrm{c}}$, and the ratio of particle to energy diffusivity in the remainder of the plasma, $D_{0}$. The radial coordinate is the square root of the normalized plasma volume.

scenarios offer strong evidence that the current profile is nonmonotonic [5], and indeed that the appearance of the barrier is tied both spatially and temporally to the appearance of a minimum in the $q$ profile $[4,7]$. This correlation has also been corroborated by transport modelling [7].

The dependence of the reconstructed $q$ profile on the details of the particle diffusivity used in CQL3D has recently been investigated [17]. Within the sole global constraint provided by the total driven current, there is considerable freedom in the choice of the radial diffusivity profile. Further constraints must be sought from other experimentally measured quantities. One plausible approach is to assume a proportionality between the particle diffusivity and the energy diffusivity inferred from power-balance calculations. As the latter is poorly constrained in the immediate proximity of the magnetic axis, where little power is deposited, the core diffusivity remains a free parameter. An alternative approach has also been explored, involving a piecewise uniform diffusivity in the three well-defined regions corresponding to the power deposition region including the barrier itself and to the spaces inside and outside it. As CQL3D simulations are performed by constraining the density profile to the experimentally measured one, the varying quantity is the electron energy; the free parameters are then adjusted heuristically to provide an equivalent electron temperature exceeding the measured bulk temperature by an amount of the order estimated for the suprathermal electron contribution. The $q$ and shear $[s=(\rho / q)(\mathrm{d} q / \mathrm{d} \rho)]$ profiles calculated for a fully noninductive discharge from a range of valid choices within the two approaches are shown in figure 4, along with the diffusivity and electron temperature profiles. The result demonstrates a remarkable resiliency of the $q$ profile from well inside the barrier $(\rho=0.35$ ) out to the plasma edge, with a noticeable variance of the (negative) shear only in the inner core, even though the variation in the calculated electron temperature is well above the experimental error bars. While the inner-core variations can be significant in detailed comparisons with theory, the primary result - that the $q$ profile is nonmonotonic - is firmly supported by this sensitivity study.

The role of the current profile has been conclusively proven by an experiment exploiting the very large current drive efficiency of the Ohmic transformer to introduce small current perturbations accompanied by negligible energy transfer. Small increases or decreases in the central current density can dramatically degrade or enhance the confinement, respectively, while the location of the barrier is largely unaffected (figure 5, where the quality of the confinement is parametrized by the barrier strength, defined as the maximum of $\rho_{T}^{*}$, the ion sound gyroradius normalized to the electron temperature gradient scale length on the outer midplane) [8]. This experiment replicates in a more controlled fashion earlier 


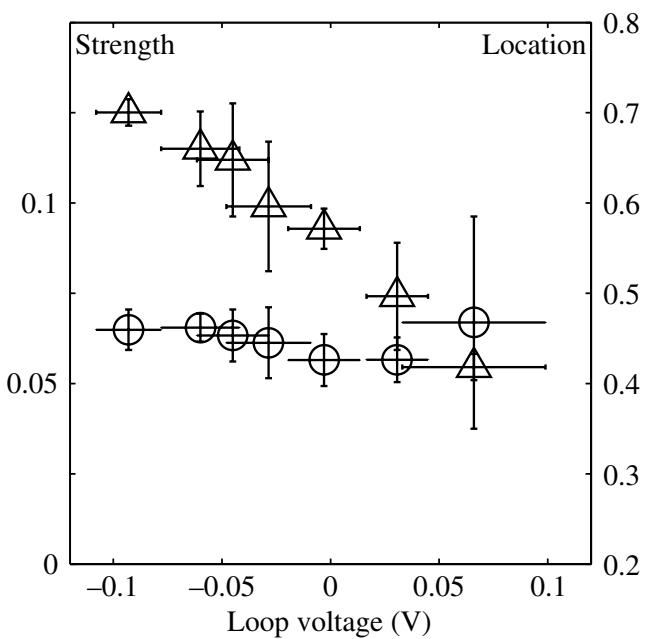

Figure 5. eITB strength (triangles: the maximum of $\rho_{T}^{*}$, the ion sound gyroradius normalized to the electron temperature gradient scale length on the outer midplane, from Thomson scattering measurements) and location (circles: radial coordinate equal to the square root of the normalized plasma volume) as functions of the surface loop voltage for a set of Ohmic current perturbation experiments in otherwise noninductive discharges. The measurements are taken in the stationary phase of the discharge after current relaxation is complete.

studies performed with varying central ECCD components [9] and confirms that a negative central $q$ shear is crucial to the creation of a barrier, with the barrier steepness and attendant confinement enhancement increasing with increasing central shear (in absolute value).

The Ohmic perturbation method, in addition, permits the observation of the transient effect of resistive current penetration into the plasma: as a positive current diffuses inwards, and before it reaches the location of maximum current density, its effect is initially to deepen the central current hole, i.e. the negative central shear becomes even more negative; only when current diffusion is complete does the shear become less steep. The opposite is true in the case of negative current injection. This transient effect is predicted by ASTRA transport simulations and has the experimentally observed effect of causing an initial enhancement or degradation of the barrier (in the cases of positive or negative injection, respectively) before the effect is reversed, as shown in figure 6 .

A series of dedicated experiments was carried out in 2005 to complete the existing TCV database of discharges with Ohmic perturbations applied to eITBs, with the aim of studying the dependence of the confinement enhancement on the perturbative loop voltage. An examination of the entire database revealed that this dependence is smooth, without any sharp variations of confinement at any particular loop voltage. It was also found that the confinement in the eventual steady-state phase did not depend on the history of how the perturbative voltage was applied (e.g. during the formation of the barrier or after the barrier was fully formed). With the aid of safety-factor profile modelling these results strongly suggested that rational $q$ values do not play a role in the formation of the barrier, at least in the range $1.3<q<2.3$ [6]. A conclusive proof would require a direct $q$-profile measurement.

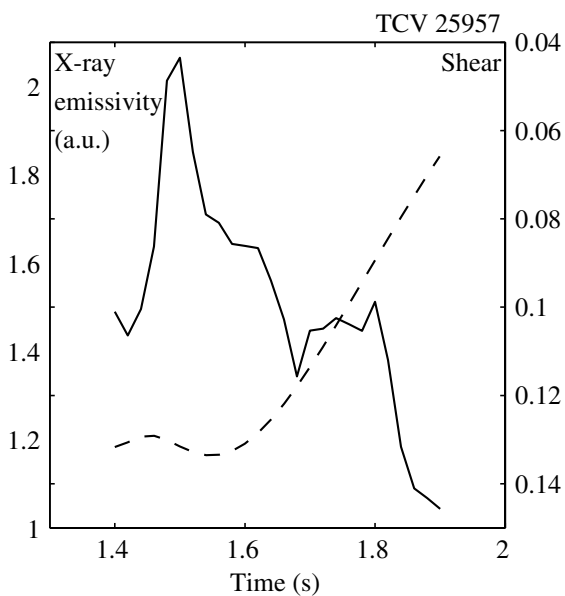

Figure 6. Central x-ray emissivity (solid curve) and magnetic shear (dashed curve), as calculated by ASTRA, averaged in the negative-shear region. The x-ray emissivity is a sensitive measure of the confinement quality, with high temporal resolution. Noninductive discharge, $65 \mathrm{mV}$ positive (co-current) loop voltage applied externally from $1.4 \mathrm{~s}$; only the initial transient phase is shown, before a stationary state is reached.

\section{The properties and dynamics of eITBs}

Barriers appear both in the electron temperature $\left(T_{\mathrm{e}}\right)$ and density $\left(n_{\mathrm{e}}\right)$ profiles. In the steady-state phase, the two barriers are strongly correlated in space and steepness, as they occur at the same location with the ratio $\eta_{\mathrm{e}}$ of the logarithmic $T_{\mathrm{e}}$ gradient to the logarithmic $n_{\mathrm{e}}$ gradient approximately equal to 2.0-2.5 [18]. While no such correlation exists in L-mode discharges, in which $\eta_{\mathrm{e}}$ varies with plasma conditions and heating characteristics, this parameter takes the asymptotic value of 2.0-2.5 in all fully developed eITBs. In particular, starting from fully noninductive conditions, the further barrier enhancement by a negative Ohmic current perturbation [8], and the attendant change in the $q$ profile, do not affect $\eta_{\mathrm{e}}$ [18].

Under the conditions of these experiments, neoclassical transport would result in a value of $\eta_{\mathrm{e}}$ close to the measured one [18]. This suggested the possibility that turbulence suppression may result in transport being reduced to neoclassical levels and motivated an experimental campaign to measure particle transport directly in these scenarios, using pulsed gas injection. These studies have yielded values for the diffusivity and convection velocity of, respectively, $0.3 \mathrm{~m}^{2} \mathrm{~s}^{-1}$ and $1 \mathrm{~m} \mathrm{~s}^{-1}$ at the barrier location; while these values are 3 to 5 times lower than their L-mode counterparts, they still exceed neoclassical transport coefficients by an order of magnitude [19]. Neoclassical transport therefore remains negligible in these discharges.

The two primary effects of turbulence on particle transport are turbulent equipartition (TEP) [20] and anomalous thermodiffusion (THD) [21]. The former, while significant in strongly heated L-mode discharges, vanishes at zero magnetic shear and is proportional to the temperature gradient scale length and thus becomes negligible in the case of a transport barrier with a reversed shear profile. The role of THD depends on the behaviour of the dominant underlying instability, which for strongly EC-heated plasmas is the trapped electron mode (TEM). This mode is strongly stabilized inside the barrier, as a 



Figure 7. (a) Growth rate and $(b)$ real frequency of the most unstable mode, as calculated by GLF23, versus minor radius for a monotonic- $q$, L-mode discharge (29863) and an eITB discharge (29866).

result of the negative magnetic shear, as shown by gyroLandau fluid simulations with the GLF23 code [22] in figure 7. However, transport is still dominated by TEM-induced THD partly because the diffusion coefficient has a maximum at very low growth rates [19] and partly because of the concomitant quenching of TEP. It can also be seen that the TEM quenching is found theoretically to occur in the region inside the barrier but not at the barrier location itself (see figure 8). As a result, the experimental value of $\eta_{\mathrm{e}}$ is fairly closely reproduced by GLF23 simulations, except in the immediate neighbourhood of the location of zero magnetic shear, where the density profile is incorrectly predicted to be flat, as shown in figure 8 . Thus, there is reason to believe that these simulations contain most of the relevant physics, but the remaining discrepancy at the location of zero shear must be addressed. One possibility to be explored by future modelling is the proper inclusion of parallel electron dynamics: up to now the parallel wave number has been set to zero. Whether this could resolve the discrepancy remains to be seen.

The dynamic evolution of an eITB after the initial, rapid inception can be quite complex. In fully noninductive
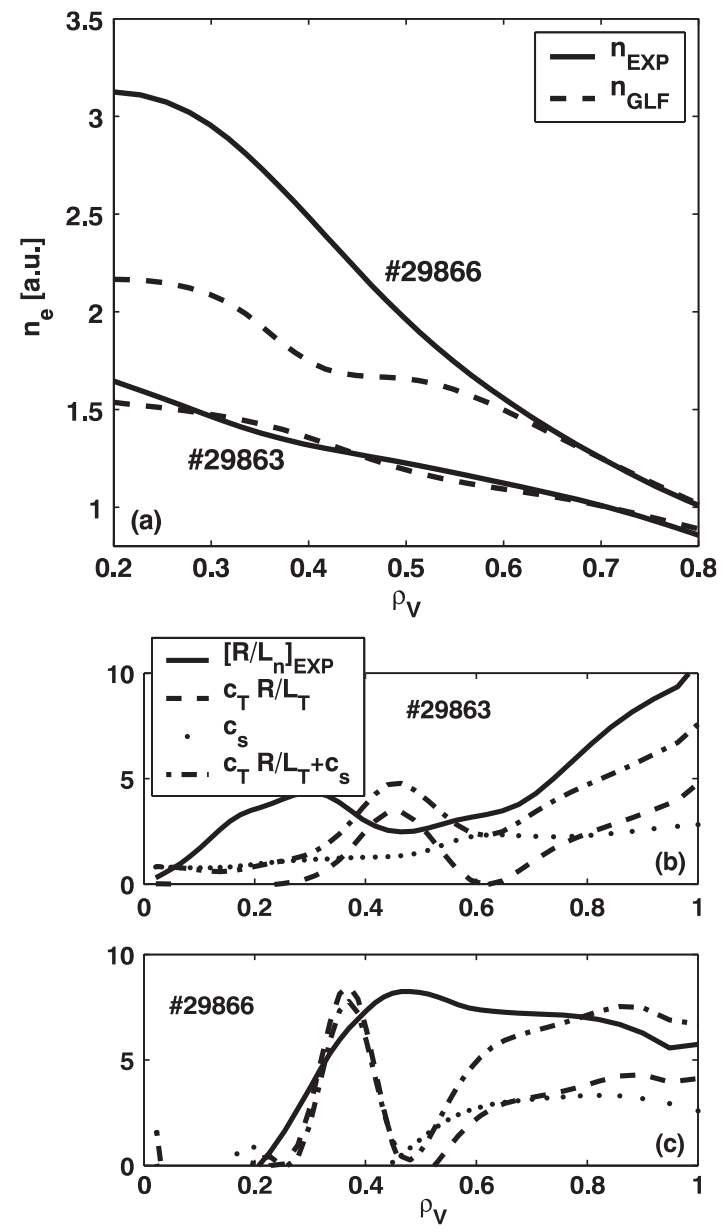

Figure 8. (a) Experimental density profiles (solid curves) versus profiles calculated by GLF23 (dashed curves) for the discharges of figure 7; $(b)$ and $(c)$ for the two discharges, experimental (solid curve) and calculated (dash-dotted curve) density gradient; the dashed and dotted curves are the TEP and THD contributions to the calculated gradient, respectively.

conditions with the barrier being generated by off-axis coECCD, a second, slower stage of barrier growth is often observed, after a delay of the order of the current redistribution time [6]. This effect is attributed to a feedback loop internal to the plasma, in which the key role is played by the bootstrap current. As the current profile relaxes after the barrier is initially formed, the relative locations of the barrier and of the heating sources can change slightly. As a result, more power can be deposited inside the high confinement region, resulting in a further increase in the gradients and thus in the bootstrap current, which is centred on the barrier and plays a dominant role in sustaining the hollow current profile. Being based on relative displacements that are even smaller than the very high aiming accuracy of the microwave beams, this feedback loop can also easily become negative, causing a deterioration of the barrier instead of an enhancement. A cyclic behaviour has also been observed in some cases, with several barrier collapses and regenerations during a single discharge. This high sensitivity to power deposition results in a certain degree of variability between nominally identical scenarios. However, the robustness of the configuration is greatly increased when a significant amount of power is deposited deliberately well 
inside the barrier in order to exploit the high confinement and optimize the overall plasma performance [6]

\section{MHD activity in eITBs}

While rational $q$ surfaces do not appear to play a role in the formation of eITBs, as discussed in section 2, the $q$ profile does affect the MHD stability of the discharge, and strong internal modes develop in some cases which can significantly degrade the confinement [5].

Slow oscillations of the electron temperature have been recently investigated in eITB scenarios, both in noninductive and inductive conditions. Although these oscillations have very low frequency $(\sim 10 \mathrm{~Hz})$ and are azimuthally and poloidally symmetric ( $m=n=0$ ) and thus are not of an MHD nature themselves, they are seen to coexist with underlying MHD modes [23,24]. A similar phenomenon has been documented on the Tore Supra tokamak, where it has been dubbed the O-regime and occurs in fully or nearly noninductive discharges with lower hybrid current drive and negative central magnetic shear [25].

An example is shown in figure 9 for a fully noninductive case. These oscillations affect the whole plasma column, as the total plasma current oscillates (with a $45^{\circ}$ phase shift with respect to the temperature oscillations) and the magnetic axis shifts radially by up to $3 \mathrm{~cm}$. An MHD mode is present and its amplitude oscillates $180^{\circ}$ out of phase with respect to the temperature. A feedback loop therefore appears to be at play, in which the MHD mode degrades the confinement, which in turn reduces the gradients and the MHD drive, so that a semi-stable oscillation can take hold. The bootstrap current fraction varies by $40-60 \%$. The mode is found to have helicity $m / n=3 / 1$ for the case of figure 9 [26], but a $2 / 1$ mode has been found to be dominant in other cases. Studies employing the Ohmic current perturbation method are underway with the aim of exploring the conditions under which a stable cyclic behaviour can occur, and particularly the dependence of this phenomenon on the details of the $q$ profile and of the barrier characteristics.

\section{Conclusions}

eITBs have been generated in TCV with ECRH in a variety of conditions, from transient to steady state and from noninductive to inductive. The energy confinement improvement over the L-mode ranges from 3 to 6 in these scenarios, which always display a high bootstrap current fraction, generally in excess of $70 \%$. A negative central shear is crucial to the formation and existence of eITBs and the confinement enhancement depends sensitively on the depth of the core current depletion, as proven by Ohmic perturbation experiments in noninductive discharges. Barriers occur in both the electron temperature and density profiles, with a fixed ratio of 2.0-2.5 between their logarithmic gradients, reproduced with satisfactory accuracy by gyrofluid simulations. Internal MHD activity can be triggered by the high barrier gradients depending on the details of the safety factor profile, resulting in some cases in a strong cyclic behaviour affecting the whole plasma column.

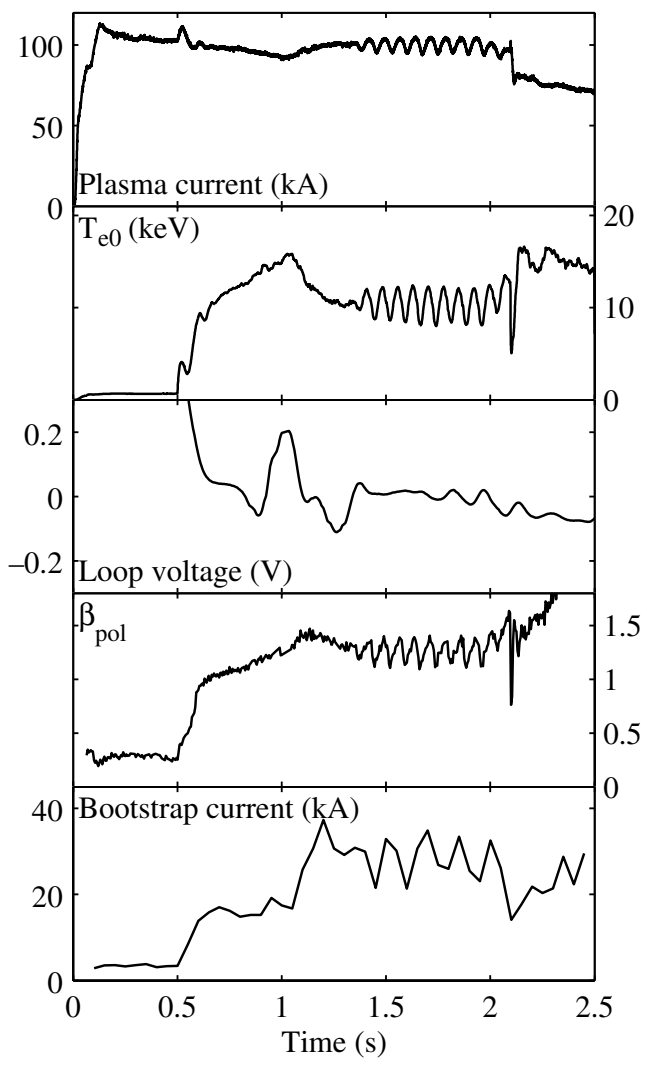

Figure 9. TCV shot 31892: plasma current, central electron temperature, loop voltage, poloidal beta, bootstrap current.

\section{Acknowledgment}

This work was supported in part by the Swiss National Science Foundation.

\section{References}

[1] Connor J.W., Fukuda T., Garbet X., Gormezano C., Mukhovatov V., Wakatani M., the ITB Database Group and the ITPA Topical Group on Transport and Internal Barrier Physics 2004 Nucl. Fusion 44 R1

[2] Wolf R.C. 2003 Plasma Phys. Control. Fusion 45 R1

[3] Hofmann F. et al 1994 Plasma Phys. Control. Fusion 36 B277

[4] Henderson M.A., Camenen Y., Coda S., Goodman T.P., Nikkola P., Pochelon A., Sauter O. and TCV Team 2004 Phys. Rev. Lett. 93215001

[5] Coda S. et al 2005 Phys. Plasmas 12056124

[6] Goodman T.P. et al 2005 Plasma Phys. Control. Fusion 47 B 107

[7] Henderson M.A., Camenen Y., Coda S., Fable E., Goodman T.P., Nikkola P., Pochelon A., Sauter O., Zucca C. and the TCV Team 2005 Nucl. Fusion 451642

[8] Sauter O. et al 2005 Phys. Rev. Lett. 94105002

[9] Henderson M.A. et al 2004 Plasma Phys. Control. Fusion 46 A275

[10] Rebut P.H., Lallia P.P. and Watkins M.L. 1989 Proc. 12th Int Conf. on Plasma Physics and Controlled Nuclear Fusion Research 1988 (Nice, 1988) vol 2 (Vienna: IAEA) $\mathrm{p} 191$

[11] Henderson M.A. et al 2003 Phys. Plasmas 101796

[12] Harvey R.W. and McCoy M.G. 1993 Proc. IAEA Technical Committee Meeting on Advances in Simulation and 
Modelling in Thermonuclear Plasmas (Montreal, 1992) (Vienna: IAEA) p 498

[13] Harvey R.W., Sauter O., Prater R. and Nikkola P. 2002 Phys. Rev. Lett. 88205001

[14] Nikkola P., Sauter O., Behn R., Coda S., Condrea I., Goodman T.P., Henderson M.A., Harvey R.W. and the TCV Team 2003 Nucl. Fusion 431343

[15] Coda S., Alberti S., Blanchard P., Goodman T.P. Henderson M.A., Nikkola P., Peysson Y. and Sauter O. 2003 Nucl. Fusion 431361

[16] Pereverzev G.V., Soldner F.X., Bartiromo R., Leuterer F. and Parail V.V. 1992 Nucl. Fusion 321023

[17] Asp E., Sauter O., Coda S., Fable E., Goodman T.P., Turri G., Udintsev V.S. and Zucca C. 2006 Theory of Fusion Plasmas (Proc. Joint Varenna-Lausanne Int. Workshop, 2006) (AIP Conference Proceedings) vol 871 p 283

[18] Fable E., Sauter O., Coda S., Goodman T.P., Henderson M.A., Weisen H., Zabolotsky A., Zucca C. and The TCV team 2006 Plasma Phys. Control. Fusion 481271
[19] Fable E., Sauter O., Marinoni A., Zucca C. and the TCV team 2006 Theory of Fusion Plasmas (Proc. Joint Varenna-Lausanne Int. Workshop, 2006) (AIP Conference Proceedings) vol 871 p 318

[20] Baker D.R. and Rosenbluth M.N. 1998 Phys. Plasmas 52936

[21] Miskane F. and Garbet X. 2000 Phys. Plasmas 74197

[22] Waltz R.E., Staebler G.M., Dorland W., Hammett G.W., Kotschenreuther M. and Konings J.A. 1997 Phys. Plasmas 42482

[23] Udintsev V.S. et al 2006 Proc. 33rd Eur. Conf. on Controlled Fusion and Plasma Physics (Rome, 2006) vol 30I (ECA) (Geneva: European Physical Society) D-1.003

[24] Udintsev V.S. et al 2007 Fusion Sci. Technol. at press

[25] Giruzzi G. et al 2003 Phys. Rev. Lett. 91135001

[26] Turri G., Sauter O., Scarabosio A., Udintsev V.S., Furno I., Zucca C. and Goodman T.P. 2006 Proc. 33rd Eur. Conf. on Controlled Fusion and Plasma Physics (Rome, 2006) vol 30I (ECA) (Geneva: European Physical Society) P-1.148 